\title{
VARIABILIDADE DA LARGURA E INTENSIDADE DA ZONA DE CONVERGÊNCIA INTERTROPICAL ATLÂNTICA: ASPECTOS OBSERVACIONAIS.
}

\author{
MIGUEL ÂNGELO VARGAS DE CARVALHO ${ }^{1}$ E MARCOS DAISUKE OYAMA ${ }^{2}$ \\ ${ }^{1}$ Instituto de Controle do Espaço Aéreo, São José dos Campos, SP, Brasil \\ ${ }^{2}$ Instituto de Aeronáutica e Espaço, São José dos Campos, SP, Brasil \\ miguelmavc@icea.gov.br, marcosoyamamdo@iae.cta.br
}

Recebido Março de 2012 - Aceito Dezembro de 2012

\begin{abstract}
RESUMO
Este trabalho apresenta um estudo observacional das características da Zona de Convergência Intertropical (ZCIT) na porção central do Oceano Atlântico. A variabilidade da posição, largura e intensidade (precipitação média) da ZCIT Atlântica durante 10 anos (1999-2008) foi obtida objetivamente de dados de precipitação pentadais. O método dos compostos foi utilizado para obter a distribuição espacial de precipitação, temperatura da superfície do mar (TSM) e ventos à superfície a fim de verificar os fatores associados à variação de largura da ZCIT. Os resultados confirmaram os de trabalhos anteriores sobre a variabilidade da posição e apresentaram informações adicionais sobre a variabilidade da intensidade e largura. Na escala mensal, a largura e intensidade da ZCIT tendem a diminuir ao atingir as suas posições mais austrais (fevereiro, março e abril). A amplitude da variação anual foi de aproximadamente $7^{\circ}$ de latitude para a posição média (de $1^{\circ} \mathrm{N}$ em abril a $8^{\circ} \mathrm{N}$ em agosto), de $3^{\circ}$ para a largura (de $3^{\circ}$ em março a $6^{\circ}$ em outubro) e, quanto à intensidade da precipitação, foi de $3 \mathrm{~mm} \cdot \operatorname{dia}^{-1}$ (de $10 \mathrm{~mm} \cdot \mathrm{dia}^{-1} \mathrm{em}$ março a $13 \mathrm{~mm} \cdot \mathrm{dia}^{-1} \mathrm{em}$ julho). Na análise dos compostos da precipitação, TSM e divergência dos ventos à superfície na região do Atlântico Tropical, encontraram-se indícios de que, em condições de posição da ZCIT próxima à média, anomalias positivas de largura estariam associadas às de negativas de TSM e às positivas de convergência dos ventos à superfície, mas não houve significância estatística das diferenças.
\end{abstract}

Palavras-Chave: ZCIT, precipitação, Oceano Atlântico, análise de compostos.

\section{ABSTRACT: ATLANTIC INTERTROPICAL CONVERGENCE ZONE WIDTH AND INTENSITY VARIABILITY: OBSERVATIONAL ASPECTS}

This work presents an observational study of the Intertropical Convergence Zone (ITCZ) features over the central portion of the Atlantic Ocean. The Atlantic ITCZ position, width and intensity (mean precipitation) variability for 10 years (1999-2008) was obtained objectively from pentad precipitation data. The composite method was used to obtain of the spatial distribution of precipitation, sea surface temperature (SST) and surface winds in order to identify the factors associated with ITCZ width variation. The results confirmed previous works on ITCZ position variability and showed additional information concerning the variability of the intensity and width. At monthly timescale, the ITCZ width and intensity tend to decrease when ITCZ reaches its southernmost positions (February, March and April). The annual variation amplitude was about $7^{\circ}$ of latitude for the mean position $\left(1^{\circ} \mathrm{N}\right.$ in April to $8^{\circ} \mathrm{N}$ in August), $3^{\circ}$ for the width ( $3^{\circ}$ in March to $6^{\circ}$ in October) and $3 \mathrm{~mm} \cdot$ day $^{-1}$ for the precipitation intensity $\left(10 \mathrm{~mm}^{- \text {day }^{-1}}\right.$ in March to $13 \mathrm{~mm}$. day ${ }^{-1}$ in July). The composite analysis of precipitation, SST and surface winds divergence over the Tropical Atlantic region showed that, under conditions of ITCZ position close to the average, positive width anomalies would be related to negative SST anomalies and positive convergence of surface winds, but the differences had no statistical significance. Keywords: ITCZ, precipitation, Atlantic Ocean, composite analysis. 


\section{INTRODUÇÃO}

A Zona de Convergência Intertropical (ZCIT) é um sistema sinótico que ocorre nas proximidades da linha do equador. Segundo Ferreira (1996), está inserida numa região onde ocorre a interação de características marcantes atmosféricas e oceânicas: i) zona de confluência dos Alísios (ZCA); ii) zona do cavado equatorial; iii) zona de máxima temperatura da superfície do mar (TSM); iv) zona de máxima convergência de massa; e v) zona da banda de máxima cobertura de nuvens convectivas, todas interagindo próximas à faixa equatorial. Apesar dessa interação as características não se apresentam, necessariamente, ao mesmo tempo, sobre a mesma latitude. A região sob a ação da ZCIT é identificada pela presença de aglomerados convectivos, um dos principais mecanismos de aquecimento da atmosfera ocasionado, principalmente, pela liberação de calor latente e pela interação entre radiação e nebulosidade. Desta forma, a compreensão do comportamento da ZCIT torna-se fundamental para o entendimento da circulação geral da atmosfera e das características do regime pluviométrico na região equatorial. Por exemplo, considera-se que a ZCIT é um dos principais sistemas que afeta a estação chuvosa no norte do nordeste brasileiro (Molion e Bernardo, 2002).

A posição média da ZCIT encontra-se um pouco ao norte do equador (Philander et al., 1996), embora a sua localização latitudinal apresente grande variação sazonal e longitudinal. Sobre o Oceano Atlântico, a ZCIT - chamada de ZCIT Atlântica - desloca-se em média de $14^{\circ} \mathrm{N}$ (agosto e setembro) a $2^{\circ} \mathrm{S}$ (março e abril). Em anos chuvosos no nordeste brasileiro (NEB), a ZCIT Atlântica pode atingir $5^{\circ} \mathrm{S}$ como posição mais austral (Melo et al., 2009). Vários estudos mostram que anomalias da TSM nos Oceanos Pacífico e Atlântico tropicais afetam o posicionamento latitudinal da ZCIT e, em particular, da ZCIT Atlântica (De Souza et al., 2005; Gu e Adler, 2009). Por exemplo, o posicionamento estaria no sentido do gradiente meridional inter-hemisférico de anomalias de TSM no Atlântico (Nobre e Shukla, 1996), ou sobre as anomalias positivas de TSM ("águas quentes") no padrão de dipolo no Atlântico (Moura e Shukla, 1981), embora a ZCIT possa estar bem definida mesmo na ausência de uma região de máxima TSM (Holton et al., 1971; Hastenrath e Lamb, 1977). A liberação de calor latente em níveis médios pode ter um papel importante na organização da convergência nos baixos níveis (Charney, 1971), produzindo, assim, algumas das características observadas do ZCIT (Waliser e Somerville, 1994). Segundo Biasutti et al. (2006), a ZCIT Atlântica tem sua localização e intensidade sobre determinadas condições ambientais, tais como, distribuição vertical de umidade e temperatura, TSM, bem como, trocas de umidade e calor entre a atmosfera e o oceano.
Embora diversos estudos sobre a ZCIT Atlântica tenham sido realizados, a sua intensidade e largura têm merecido menos atenção na literatura do que a sua posição latitudinal. A intensidade, segundo Xie e Carton (2004), é mais fraca em março e abril que em julho e agosto, apesar do aumento de $1^{\circ} \mathrm{C}$ da TSM no Atlântico durante o outono austral. No sentido longitudinal, a ZCIT é notadamente mais intensa na região central do Atlântico, do que perto da costa da América do Sul de meados de julho até meados de dezembro (Kousky, 1988). Por sua vez, Melo et al. (2009) mostra que a convecção mais intensa se situa no Hemisfério de verão: mais próxima da costa do Brasil em janeiro e fevereiro, e mais próxima da costa da África entre julho e setembro. Nos meses de março a junho, a convecção se estende sobre o Atlântico Tropical de uma costa a outra, embora os máximos fiquem concentrados próximos às duas costas. De outubro a dezembro, os máximos situam-se na região central do Atlântico Tropical.

Em relação à largura, os estudos que abordam a sua variabilidade temporal e espacial são relativamente escassos e pouco conclusivos. Segundo Khrgian (1977), no Hemisfério Norte (HN), a ZCIT é mais larga (200-600 km) e regular do que no Hemisfério Sul (HS) ( $\sim 300 \mathrm{~km})$, onde frequentemente apresenta-se quebrada. From e Staver (1979) afirmam que varia entre 20 milhas $(\sim 30 \mathrm{~km})$ a 300 milhas $(\sim 480 \mathrm{~km})$. Segundo Das (1986), a ZCIT é uma região estreita em torno de 200-300 km.

Como uma etapa preparatória para abordar a questão das anomalias de precipitação em algumas regiões, como o norte do NEB, com o papel das anomalias da posição, largura e precipitação média (intensidade) da ZCIT (PLPMZ), o objetivo deste trabalho é realizar um estudo observacional climatológico da PLPMZ sobre a região central do Oceano Atlântico. Para obter a PLPMZ, implementa-se um método objetivo diferente dos utilizados em estudos anteriores (Kousky, 1988; Uvo, 1989; Hastenrath, 1990), principalmente quanto à estimativa de sua intensidade e largura. Especificamente para a largura, objetiva-se também verificar se as suas anomalias estão associadas às de TSM ou de convergência atmosférica à superfície, nos meses em que a ZCIT atinge a sua posição mais austral (seguindo metodologia semelhante a Chen et al., 2008). A relação entre as anomalias de PLPMZ e as de precipitação será abordada em trabalhos futuros.

\section{MATERIAL E MÉTODOS}

Totais diários de precipitação da versão 1.1 do Global Precipitation Climatology Project (GPCP) são utilizados no método objetivo para identificar as características da ZCIT. Os dados estão dispostos em uma grade regular com espaçamento de $1^{\circ}$ cobrindo todo o globo e estão disponíveis para o período de outubro de 1996 a junho de 2009. Os totais diários de 
precipitação são estimados a partir de produtos de satélites no canal infravermelho, microondas e sondagens do Television and Infrared Observation Satellite (TIROS) Operational Vertical Sounder (TOVS) e, posteriomente, são ajustados com as análises da versão 2 do GPCP mensal (Huffman et al., 2001). Os dados são arquivados e distribuídos através do World Data Center A do National Oceanic and Atmospheric Administration (NOAA) e podem ser acessados pela internet ${ }^{1}$.

São utilizados também os dados diários de TSM do Tropical Rainfall Meassuring Mission Microwave Imager (TMI) e de ventos à superfície sobre o oceano do escaterômetro SeaWinds para estudar a correlação entre essas variáveis e a PLPMZ. Esses dados são disponibilizados pelo Remote Sensing Systems na internet ${ }^{2}$. Os dados de TSM estão disponíveis de dezembro de 1997 até o presente; os de ventos à superfície sobre o oceano, de julho de 1999 a novembro de 2009. Os dados diários de TSM estão dispostos em uma grade regular com espaçamento de $0,25^{\circ}$ com cobertura de $40^{\circ} \mathrm{S}$ a $40^{\circ} \mathrm{N}$. As estimativas de TSM pelo TMI são obtidas de um algoritmo que ajusta as temperaturas de brilho ao modelo de transferência radiativa que é uma função da TSM, velocidade do vento, vapor d'água e água líquida das nuvens (Simpson et al., 1988; Kummerow et al., 1998). Os dados diários de ventos à superfície estão dispostos em uma grade regular com espaçamento de $0,25^{\circ}$ com cobertura de $89,875^{\circ} \mathrm{S}$ a $89,875^{\circ} \mathrm{N}$. O satélite QuikBird foi lançado pelo National Aeronautics and Space Administration (NASA) em junho de 1999. A bordo desse satélite estão os instrumentos SeaWinds, também conhecido como QuikScat. O QuikScat é um escaterômetro de ondas curtas que mede a direção e velocidade do vento a $10 \mathrm{~m}$ de altura da superfície do oceano (Hoffman e Leidner, 2005).

A área escolhida para identificar e estudar a ZCIT estende-se entre as latitudes $10^{\circ} \mathrm{S}$ e $18^{\circ} \mathrm{N}$ e as séries de dados são analisadas ao longo da longitude de $27,5^{\circ} \mathrm{W}$. Esta longitude, correspondente à região central do Oceano Atlântico Tropical, representa melhor a estrutura da ZCIT, ao diminuir a influência da atividade convectiva transiente dos continentes africano e sul-americano (Chiang et al., 2002). Para se estimar a PLPMZ entre os anos de 1999 a 2008 (10 anos), são utilizados totais diários de precipitação do Global Precipitation Climatology Project (GPCP). A resolução dos dados de precipitação $\left(1^{\circ}\right)$ é mais adequada para análise da PLPMZ, principalmente a sua largura. Com esses dados, são calculadas médias de 5 dias (pêntadas) com o objetivo de eliminar as flutuações diárias associadas aos distúrbios de alta frequência. A PLPMZ é obtida objetivamente da variação meridional de dados de precipitação pentadais. Basicamente, o método objetivo consiste em analisar

1. http://www1.ncdc.noaa.gov/pub/data/gpcp/1dd-v1.1

2. ftp://ftp.ssmi.com as regiões ao longo da longitude de $27,5^{\circ} \mathrm{W}$ com valores de precipitação acima do limiar de aproximadamente $7 \mathrm{~mm} \cdot \mathrm{d}^{-1}$. Esse limiar, condizente com o adotado na literatura (Legates e Willmott, 1990), leva à caracterização da ZCIT (posição e largura) comparável à obtida utilizando dados de baixa resolução (espaçamento de $2,5^{\circ}$ em grade regular) de radiação de onda longa emergente (ROLE) (p. ex., Kousky, 1988; Liebmann et al., 1999; Lau et al., 1997; Ferreira et al., 2005).

A Equação 1 determina a posição latitudinal média da ZCIT (na longitude de $27,5^{\circ} \mathrm{W}$ ) durante a pêntada $\mathrm{k}$, segundo a expressão:

$$
\overline{l a t_{i}}=\frac{\sum \text { lat }_{i} . w_{i}}{\sum w_{i}}
$$

onde $l a t_{i}$ é a latitude dos pontos de grade em que a precipitação é maior do que o limiar, e $w_{i}$ é o peso, calculado como a razão entre a precipitação e o limiar. O uso de média ponderada difere de outros métodos, tais como, de associar a posição da ZCIT ao mínimo de ROLE (Kousky, 1988; Ferreira et al., 2005) ou temperatura de brilho no canal do infravermelho (Pimentel e Ferreira, 1992), mas permite que a posição seja uma medida global, e não local, dos pontos com precipitação suficientemente alta.

A largura da ZCIT é definida como o a distância entre a $l a t_{i}$ mais ao norte e a $l a t_{i}$ mais ao sul, que contém a posição e cujos valores de precipitação são maiores do que o limiar durante uma determinada pêntada $\mathrm{k}$. A intensidade corresponde à precipitação média observada no intervalo de largura da ZCIT durante a pêntada k. Uma descrição detalhada do método objetivo utilizado para identificar a PLPMZ encontra-se no Apêndice.

Os resultados são caracterizados estaticamente, com o intuito de apresentar e discutir o comportamento da PLPMZ. A análise estatística considera todos os dados disponíveis (em pêntadas), mas os resultados são apresentados na escala mensal. As pêntadas são agrupadas mensalmente (6 pêntadas), num total de 72 distribuídas ao longo do ano. Portanto, a última "pêntada" de cada mês pode ter de 3 a 6 dias, conforme o número de dias do mês (Uvo, 1989).

O método dos compostos é utilizado para obter a distribuição espacial de precipitação, TSM e ventos à superfície na região do Atlântico Tropical, com a finalidade de verificar os fatores associados às anomalias de largura da ZCIT. Os dados de precipitação utilizados para confecção dos compostos são provenientes dos totais diários de precipitação do GPCP. Os dados diários de TSM são obtidos do TRMM TMI. Os dados de ventos à superfície sobre o oceano provêm do escaterômetro SeaWinds do satélite QuikBird (QuikScat). A distribuição dos dados de TSM e vento, associados às órbitas individuais de seus respectivos satélites, geram mapas incompletos. A escolha da escala temporal pentadal (5 dias) minimiza este problema, 
pois é possível compor uma única figura com a média calculada dos dados de até 10 órbitas. O período analisado consiste nos meses de março e abril (2000-2008), quando a ZCIT atinge a sua posição mais austral. Nesse período, os dados estão disponíveis para os três conjuntos de variáveis (precipitação, TSM e ventos oceânicos à superfície).

A metodologia utilizada para confecção dos compostos é semelhante à utilizada por Chen et al. (2008). Inicialmente, selecionam-se os eventos (as pêntadas) em que a posição da ZCIT se encontra entre $\mu \pm 1,5 \cdot \delta$ (onde $\mu$ é a média e $\delta$ o desvio padrão das médias pentadais), ou seja, os eventos com grandes desvios da posição em relação à média são descartados. Os eventos selecionados são divididos em três categorias: larguras grandes (LGZ), em que a largura é superior a $\mu+\delta$; larguras pequenas (LPZ), em que a largura é inferior a $\mu-\delta$; e larguras normais, cujo módulo da diferença em relação à média é inferior a $\delta$. Os compostos são feitos para as categorias LGZ e LPZ, ou seja, filtram-se as larguras normais. Assim, as categorias abrangem os eventos "normais" em relação à posição, mas com larguras muito distintas (larguras "muito" grandes, LGZ, e larguras "muito" pequenas, LPZ). Os eventos em cada categoria são utilizados para confeccionar os compostos espaciais de precipitação, TSM e ventos à superfície sobre o oceano. A significância estatística da diferença entre os compostos (LGZ e LPZ) é avaliada pelo teste $t$ de Student (p.ex. Panofsky e Brier, 1968; Spielgel, 1979):

$$
\begin{gathered}
t=\frac{\bar{X}_{1}-\bar{X}_{2}}{\sigma \sqrt{1 / N_{1}+1 / N_{2}}} \\
\sigma=\sqrt{\frac{N_{1} \delta_{1}^{2}+N_{2} \delta_{2}^{2}}{N_{1}+N_{2}-2}}
\end{gathered}
$$

onde $N_{1}$ e $N_{2}$ são, respectivamente, os números de eventos de LGZ e LPZ $\left(N_{l}=8 ; N_{2}=6\right)$ e $\bar{X}_{1}, \bar{X}_{2}, \delta_{1}$ e $\delta_{2}$ são as médias e os desvios padrões das duas amostras. A distribuição $t$ de Student tem $v=N_{1}+N_{2}-2$ graus de liberdade. Para o nível de significância de 5\% com 12 graus de liberdade, o valor de $t$ é de 2,18 .

\section{RESULTADOS E DISCUSSÃO}

Utilizando o método objetivo, foi estimada a variabilidade mensal da PLPMZ ao longo da longitude de $27,5^{\circ} \mathrm{W}$ para as pêntadas de 1999 a 2008 (10 anos). As regiões sob altos índices de precipitação com valores acima do limiar de precipitação $\left(\sim 7 \mathrm{~mm} . \mathrm{d}^{-1}\right)$ representam a ZCIT. A Figura 1 mostra as médias mensais de janeiro de 1999 a dezembro de 2008 (10 anos) da posição (a), largura (b) e precipitação média (c) estimada da ZCIT. As barras verticais representam os desvios padrões. Na
Figura 1a, percebe-se que o ciclo anual de migração da ZCIT corresponde ao obtido em estudos anteriores (p. ex. Hastenrath e Heller, 1977; Uvo, 1989). As posições mais austrais ocorreram nos meses de fevereiro, março e abril, e as mais boreais, em julho, agosto e setembro. A amplitude da variação meridional média da posição da ZCIT, considerando valores mensais, foi de aproximadamente $7^{\circ}$ (de $1^{\circ} \mathrm{N}$ em abril a $8^{\circ} \mathrm{N}$ em agosto).

$\mathrm{Na}$ Figura 1b, foram observados a ocorrência dos valores mínimos da largura em janeiro, fevereiro e março e os máximos em outubro e novembro. De abril a setembro, a largura foi aproximadamente constante $\left(5 \pm 0,5^{\circ}\right)$. A amplitude da variação média da largura, considerando valores mensais, foi de aproximadamente $3^{\circ}$ (de $3^{\circ} \mathrm{em}$ março a $6^{\circ}$ em outubro). Estes resultados estão próximos aos encontrados por Khrgian (1977), que mostrou uma variação de largura entre 200 e 600 km.

(a)
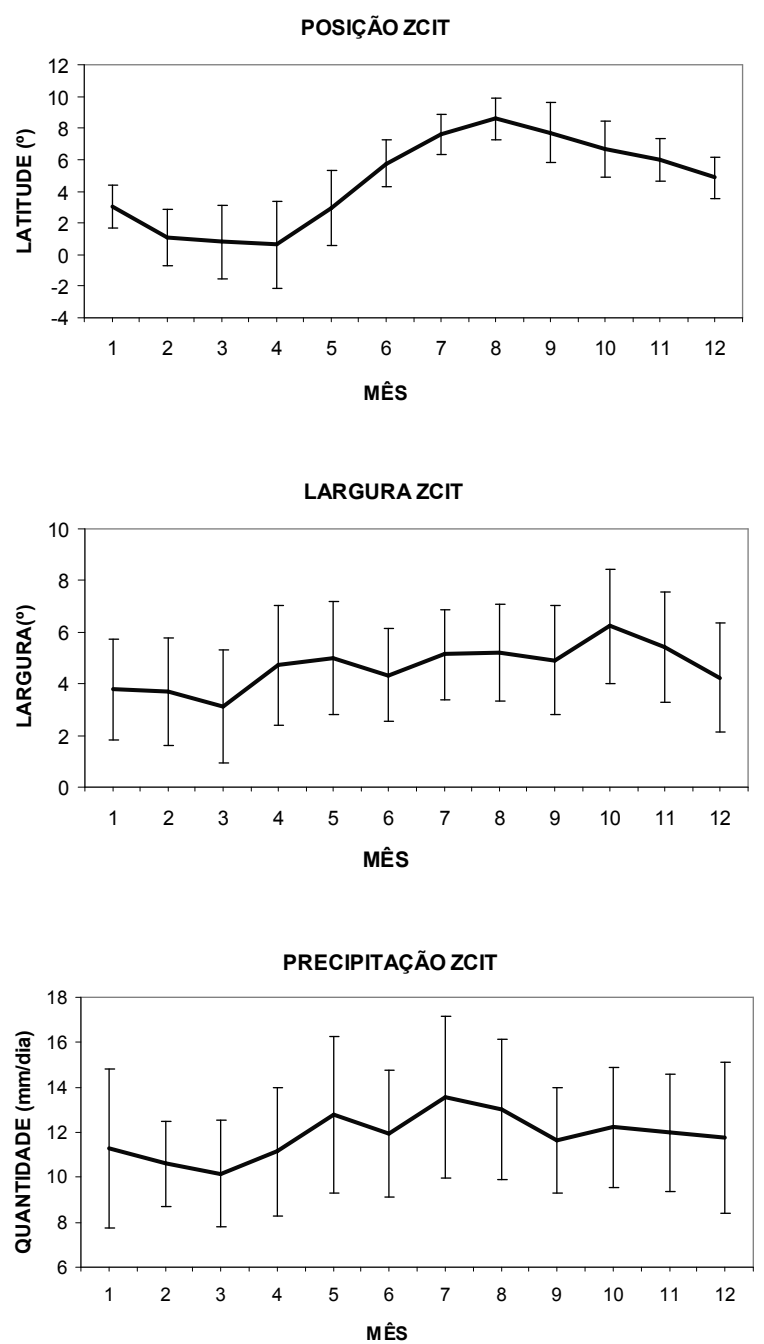

Figura 1 - Variabilidade mensal média da posição latitudinal (a), largura (b) e precipitação média (intensidade) (c) estimada da ZCIT na longitude de $27,5^{\circ} \mathrm{W}$. As barras verticais representam o desvio-padrão. 
$\mathrm{Na}$ Figura 1c, verifica-se que a menor quantidade de precipitação média ocorre em fevereiro e março; a maior, entre maio e agosto. A amplitude da variação média da precipitação da ZCIT, considerando valores mensais, foi de aproximadamente $3 \mathrm{~mm} \cdot \mathrm{dia}^{-1}$ (de $10 \mathrm{~mm} \cdot \mathrm{dia}^{-1}$ em março a $13 \mathrm{~mm} \cdot \mathrm{dia}^{-1} \mathrm{em}$ julho). Resultados semelhantes foram descritos por Xie e Carton (2004).

Duas características interessantes foram encontradas no período em que a ZCIT atinge as suas posições mais austrais (março e abril). Nesse período, a ZCIT apresenta-se mais estreita (menor largura) e menos intensa (menor precipitação média), e os desvios padrões da largura e posição são máximos (Tabela 1). Conforme a literatura (Uvo, 1989; Coelho, 2002), essa grande variabilidade pode estar associada a diferentes sistemas transientes de grande escala que atuam nos continentes sulamericano/africano (distúrbios ondulatórios de leste, vórtices ciclônicos em altos níveis, etc.) e afetam a ZCIT.

De forma semelhante à metodologia proposta por Chen et al. (2008), o método dos compostos é utilizado para se obter os campos espaciais de precipitação, TSM e divergência dos ventos à superfície na região do Atlântico Tropical. A Figura 2 mostra os compostos de precipitação do GPCP associados aos eventos de LPZ (Figura 2a, composto 1) e LGZ (Figura 2b, composto 6), a diferença entre os compostos 6 e 1 (Figura 2c) e o cálculo da significância estatística pelo teste $t$-Student (Figura 2d). Observou-se que o composto 1, associado à LPZ (Figura 2a), é caracterizado por uma banda zonal de precipitação localizada entre aproximadamente $2^{\circ} \mathrm{S}$ e $4^{\circ} \mathrm{N}$. A precipitação média variou de 5 a $12,5 \mathrm{~mm} \cdot \mathrm{dia}^{-1}$, com pico máximo concentrando-se em torno de $1^{\circ} \mathrm{N}$. Os eventos de LPZ ocorreram em $43 \%$ dos 14 selecionados. O composto 6 , associado aos eventos de LGZ (Figura $2 b$ ), ocorreu com maior frequência (57\%). Apresentou como característica mais relevante a sua inclinação no sentido SW-NE, estendendo sobre praticamente toda a extensão longitudinal do Atlântico Equatorial, desde o norte do NEB até o continente africano. A faixa de precipitação média localizou-se em sua grande parte entre $4^{\circ} \mathrm{S}$ e $4^{\circ} \mathrm{N}$, variando de 5 a $20 \mathrm{~mm} \cdot \mathrm{dia}^{-1}$. A Figura $2 \mathrm{c}$ mostra a distribuição de precipitação resultante da diferença entre os compostos 6 (LGZ) e o 1 (LPZ). As maiores anomalias positivas de precipitação coincidem com os valores significativos no nível de confiança de $95 \%$. Esta região tem início próximo à costa norte do NE e se estende até a longitude de $22^{\circ} \mathrm{W}$, entre aproximadamente as latitudes de $3^{\circ} \mathrm{S}$ e o equador. Entre $1^{\circ} \mathrm{Ne} 8^{\circ} \mathrm{N}$, percebe-se outra região com menor inclinação em torno das longitudes de $28^{\circ} \mathrm{W}$ e $12^{\circ} \mathrm{W}$. Uma característica interessante é que a largura aumenta preferencialmente em direção ao sul (Figuras 2c e 2d).

A Figura 3 é semelhante à Figura 2 e se refere às composições médias de TSM $\left({ }^{\circ} \mathrm{C}\right)$. Os compostos mostram ambos os eventos (LPZ e LGZ) localizados dentro das regiões que apresentam valores de TSM maiores que $27^{\circ} \mathrm{C}$, sugerindo que esses dois padrões da ZCIT estão intimamente associados ao ciclo anual de migração meridional da TSM do Atlântico. A Figura $3 \mathrm{c}$ mostra uma extensa região longitudinal, numa faixa latitudinal entre $1^{\circ} \mathrm{S}$ e $5^{\circ} \mathrm{N}$, de anomalias positivas de TSM entre duas regiões de anomalias negativas TSM. As anomalias positivas de TSM encontram-se ligeiramente ao norte das áreas onde se observam as maiores diferenças de precipitação entre os compostos 6 e 1 (Figura 2c). Um aspecto interessante é que a expansão da largura ocorre sobre águas mais frias. Os resultados, no entanto, mostram somente pequenas regiões com significância estatística (Figura 3d).

A Figura 4 é semelhante à Figura 2 e se refere às composições médias de divergência dos ventos à superfície $\left(10^{-5} \mathrm{~s}^{-1}\right)$. As Figuras $4 \mathrm{a}$ e $4 \mathrm{~b}$ mostram uma extensa região de convergência (divergência negativa), próxima ao equador, semelhante à distribuição de precipitação em ambos os eventos

Tabela 1 - Desvios padrões para posição, largura (em graus) e intensidade (em mm.dia ${ }^{-1}$ ) da ZCIT. Os meses em negrito representam o período com os maiores valores de desvio padrão para a largura e posição.

\begin{tabular}{c|c|c|c}
\hline \multicolumn{4}{|c}{ Desvios Padrões } \\
\hline Mês & Posição & Largura & Intensidade \\
\hline Janeiro & 1,36 & 1,95 & 3,55 \\
\hline Fevereiro & 1,80 & 2,09 & 1,89 \\
\hline Março & 2,36 & 2,21 & 2,36 \\
\hline Abril & 2,75 & 2,32 & 2,84 \\
\hline Maio & 2,35 & 2,18 & 3,47 \\
\hline Junho & 1,48 & 1,80 & 2,81 \\
\hline Julho & 1,27 & 1,75 & 3,59 \\
\hline Agosto & 1,32 & 1,89 & 3,13 \\
\hline Setembro & 1,88 & 2,11 & 2,33 \\
\hline Outubro & 1,78 & 2,20 & 2,68 \\
\hline Novembro & 1,38 & 2,14 & 2,60 \\
\hline Dezembro & 1,30 & 2,10 & 3,36
\end{tabular}




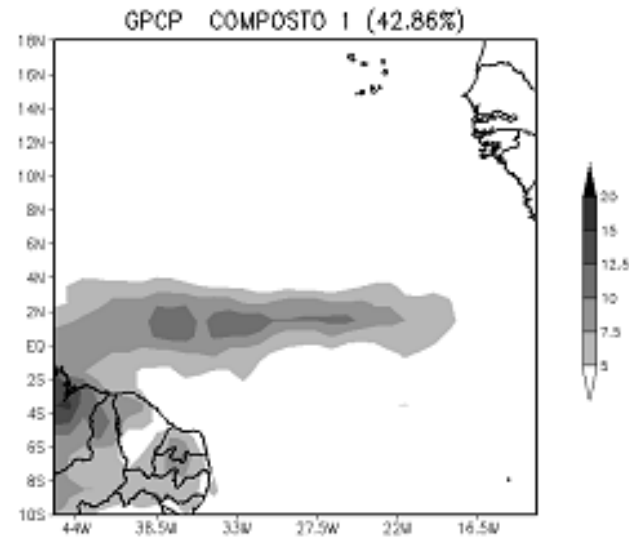

(a)

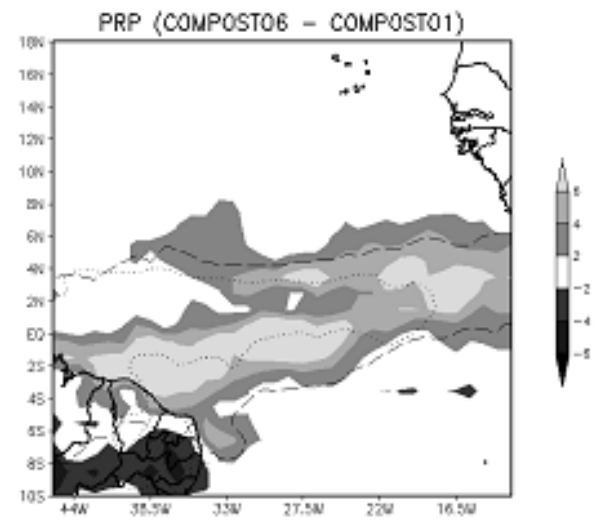

(c)

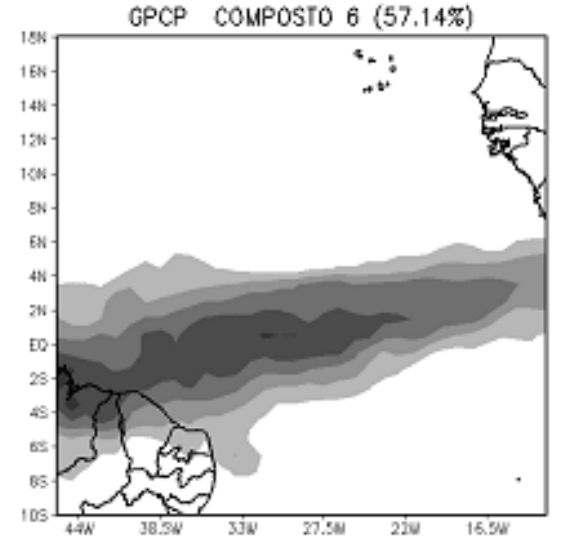

(b)

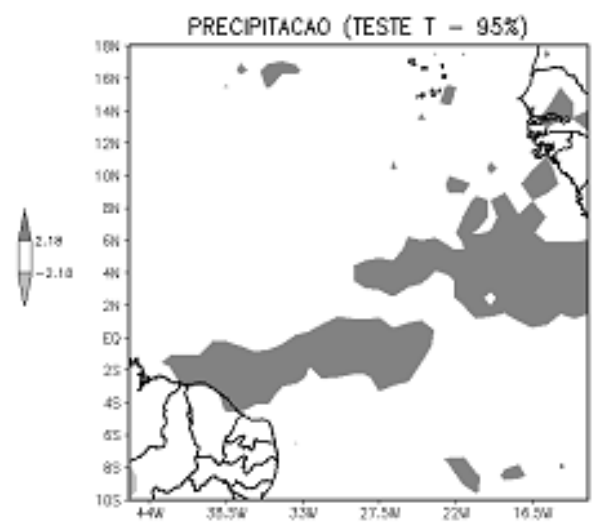

(d)

Figura 2 - Composições médias de precipitação ( $\mathrm{mm}_{\text {.dia }}{ }^{-1}$ ) associadas aos eventos de LARGP (a) e LARGG (b) para os meses de março e abril (2000-2008), diferenças entre os compostos (c) e teste de significância (d). Sombreados indicam valores significativos no nível de confiança de 95\%. A linha pontilhada (tracejada) indica a projeção do composto de precipitação 1 (6).

(LPZ e LGZ). Nota-se, no composto 8, associado aos eventos de LGZ, por exemplo, uma ampliação latitudinal da área de convergência, à medida que se aproximou do NEB. Desta forma, a área de convergência associada aos eventos de LGZ torna-se muito semelhante à área projetada pelo composto 6 . Na Figura $4 \mathrm{c}$, a diferença entre a divergência dos ventos à superfície, entre os compostos 8 e 3, mostra uma região de divergência positiva confinada por regiões de divergência negativa (convergência). Nota-se que a expansão de largura ocorre sobre as regiões de convergência. Entretanto, novamente não se encontrou significância estatística (Figura 4d).

Considerando conjuntamente os resultados, observa-se que o aumento de largura ocorre sobre águas mais frias e sob maior convergência dos ventos à superfície. Confirmando estudos anteriores (Waliser e Somerville, 1994; Biasutti et al., 2006; Chen et al., 2008, Gu e Adler, 2009), as regiões de máxima precipitação nem sempre correspondem as de anomalias positivas de TSM. Por sua vez, os resultados indicam o importante papel da interação entre a convergência nos baixos níveis e a liberação de calor latente nos níveis médios (feedback positivo). Segundo Waliser e Somerville (1994), esse seria o principal mecanismo para o desenvolvimento e manutenção da ZCIT Atlântica em algumas latitudes "preferenciais".

A Figura 5 mostra, através de gráficos de dispersão, as categorias dos compostos de precipitação em função das categorias dos compostos de DIV (a) e TSM (b). Percebe-se a tendência das categorias dos compostos de precipitação diminuir à medida que as categorias dos compostos de divergências tornaram-se menores (Figura 5a). Isto ocorre, tanto para os eventos associados à LPZ, quanto para LGZ. Por sua vez, apesar do gráfico de dispersão apresentar uma linha de tendência, nota-se uma correlação muito fraca entre as categorias dos compostos de precipitação e os compostos 


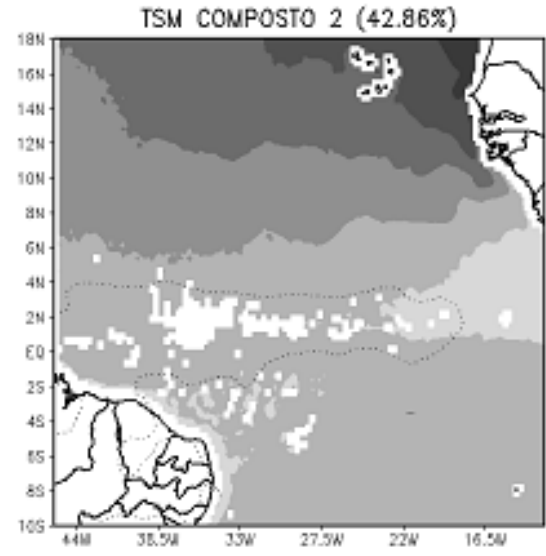

(a)

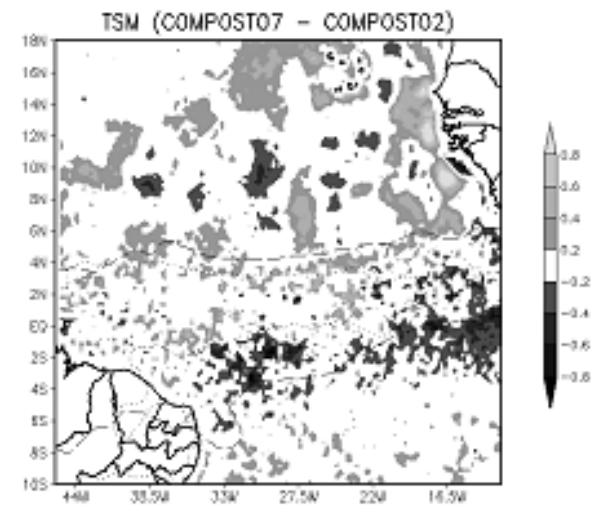

(c)

Figura 3 - Idem à Figura 2, para composições médias de TSM $\left({ }^{\circ} \mathrm{C}\right)$.

de TSM (Figura 5b). Este comportamento pode ser confirmado pelos coeficientes de determinação $\left(\mathrm{R}^{2}\right)$, mostradas na área de plotagem (canto superior direito) da Figura 5. O $\mathrm{R}^{2}$, utilizando as categorias dos compostos de DIV como variável independente, foi de 0,143 . Utilizando as categorias dos compostos de TSM, o $\mathrm{R}^{2}$ foi de 0,002 . Percebe-se ainda que a dispersão das categorias dos compostos de TSM parece ser maior para os seus valores mais altos $\left(>28,4^{\circ} \mathrm{C}\right)$.

As altas temperaturas da TSM podem ter influenciado os baixos valores correlacionados com a precipitação. Estudos anteriores mostraram a fraca influência da TSM sobre a convecção tropical ao atingir valores maiores do que $28^{\circ} \mathrm{C}$ (Gadgil et al., 1984; Gutzler e Wood, 1990). Segundo Graham e Barnett (1997), TSM acima de $27,5^{\circ} \mathrm{C}$ são necessárias para a ocorrência da convecção, entretanto não é uma condição suficiente para explicar todas as suas características. Os autores concluem que, quando as TSM estão acima de $27,5^{\circ} \mathrm{C}$, a divergência dos ventos superficiais está intimamente associada à presença ou ausência da convecção profunda.

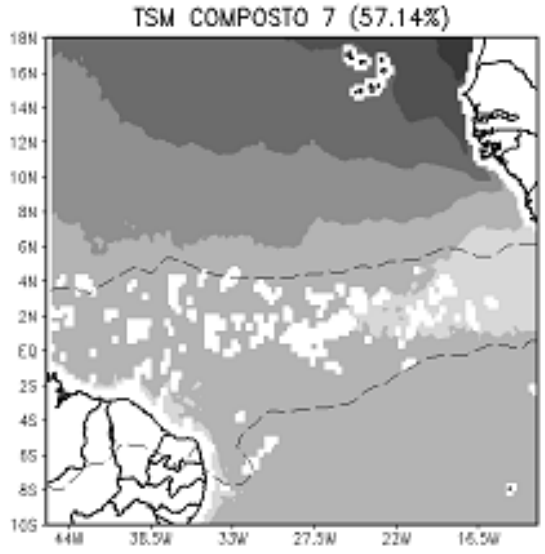

(b)

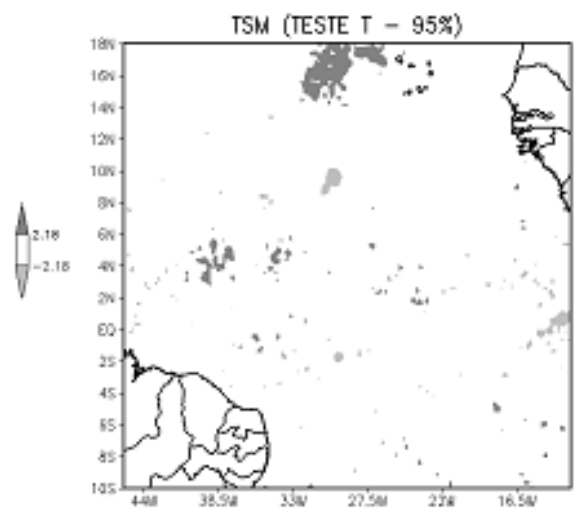

(d)

\section{COMENTÁRIOS FINAIS}

O presente estudo investigou o comportamento temporal e espacial das características da ZCIT (posição, largura e intensidade - PLPMZ) sobre a região central do Oceano Atlântico (longitude de $27,5^{\circ} \mathrm{W}$ ). A PLPMZ foi estimada objetivamente utilizando totais pentadais de precipitação para um período de 10 anos (1999 a 2008). O método objetivo difere dos utilizados em estudos anteriores (Kousky, 1988; Uvo, 1989; Hastenrath, 1990), principalmente quanto à estimativa de sua intensidade e largura. Além disso, o espaçamento de grade dos dados $\left(1^{\circ}\right)$ permite uma caracterização adequada da largura da ZCIT e, consequentemente, da intensidade, medida aqui como a precipitação média no intervalo da largura da ZCIT. O limiar de precipitação escolhido foi de aproximadamente 7 $m m . d^{-1}$ para delinear a região de atuação da ZCIT Atlântica. $\mathrm{O}$ estudo observacional confirmou estudos anteriores sobre a variabilidade da posição e apresentou informações adicionais sobre a variabilidade da intensidade e largura. 


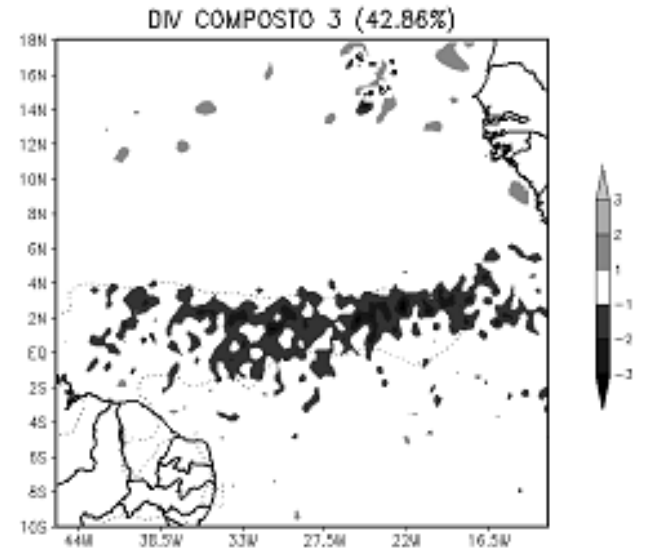

(a)

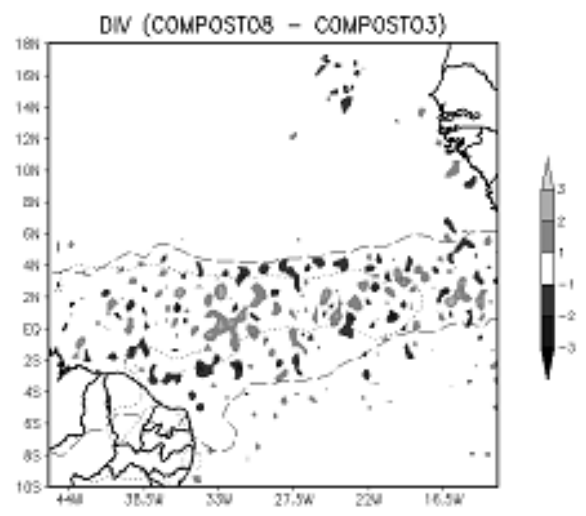

(c)

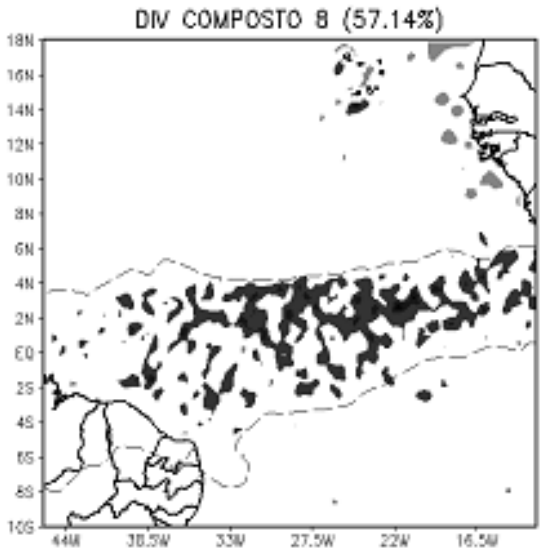

(b)

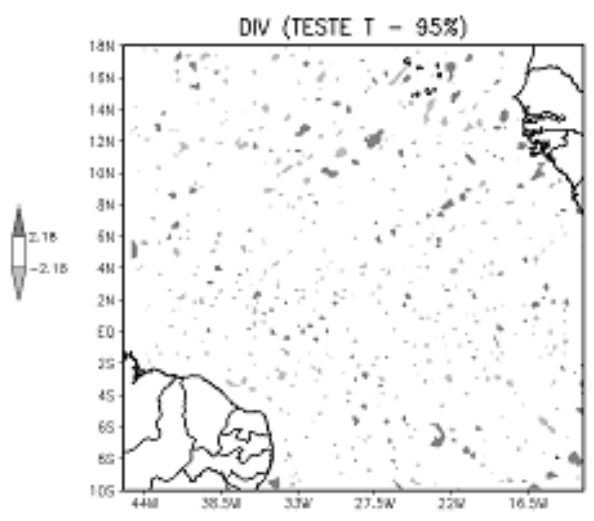

(d)

Figura 4 - Idem à Figura 2, para composições médias de divergência $\left(10^{-5} \mathrm{~s}^{-1}\right)$.

$\mathrm{Na}$ escala mensal, os resultados mostraram que a largura e intensidade da ZCIT tendem a diminuir ao atingir as suas posições mais austrais (fevereiro, março e abril). A amplitude da variação anual foi de aproximadamente $7^{\circ}$ para a posição $\left(\right.$ de $1^{\circ} \mathrm{N}$ em abril a $8^{\circ} \mathrm{N}$ em agosto), $3^{\circ}$ para a largura (de $3^{\circ}$ em março a $6^{\circ}$ em outubro) e $3 \mathrm{~mm} \cdot$ dia $^{-1}$ para a intensidade (de 10 a $13 \mathrm{~mm} \cdot \mathrm{dia}^{-1}$ em julho). Quando as posições mais austrais são atingidas, também se nota um aumento do desvio padrão, tanto da largura quanto da posição, o que sugere uma maior ação de sistemas transientes de grande escala não somente sobre os continentes sul-americano/africano, mas também sobre a ZCIT Atlântica.

Por meio da análise dos compostos de precipitação, foram estudadas as relações entre as variações da PLPMZ e as variações de TSM e divergência dos ventos à superfície. Encontraram-se indícios (pois não houve significância estatística) de que anomalias positivas de largura estariam associadas a menor TSM e maior convergência dos ventos à superfície. Um período maior de dados (30 anos, por exemplo) seria necessário para testar os indícios encontrados de forma mais completa. Através de gráficos de dispersão, nota-se uma correlação forte (fraca) entre as categorias dos compostos de precipitação e os compostos de DIV (TSM) nos meses de março e abril. Os resultados confirmam estudos anteriores sobre a importância da divergência dos ventos superficiais para a convecção tropical, quando as TSM estão acima de $27,5^{\circ} \mathrm{C}$ (p.ex. Graham e Barnett, 1997).

Espera-se que o presente trabalho contribua para uma melhor compreensão das características associadas à ZCIT. Informação detalhada e precisa do comportamento desse sistema é necessária para previsão de tempo e clima, tornando-se útil para serem aplicadas no aperfeiçoamento da previsão de chuvas na estação chuvosa das regiões norte e nordeste do Brasil. Semelhantes a estudos realizados para o posicionamento da ZCIT, como trabalhos futuros sugerem-se estudar a relação entre as variabilidades da sua largura e intensidade com as de precipitação para as regiões norte e nordeste do Brasil, bem como, a sua relação com fatores como El Niño-Oscilação Sul (ENOS) e gradiente inter-hemisférico do Atlântico (GRAD). 
(a)

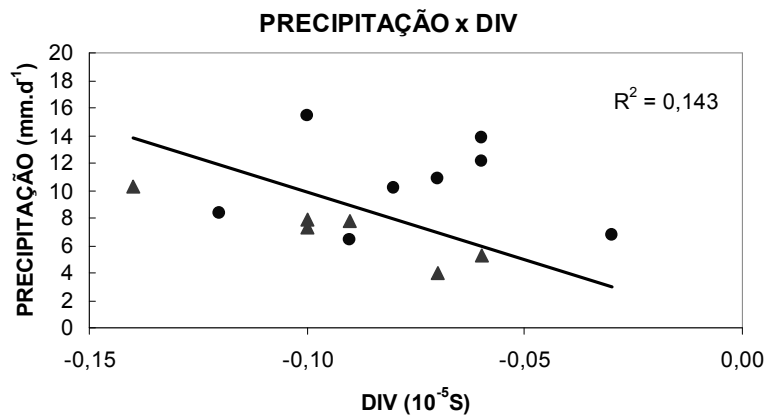

(b)

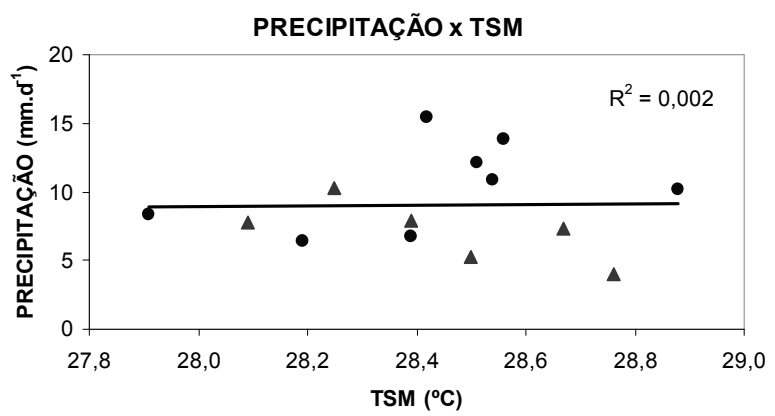

Figura 5 - Gráficos de dispersão para as categorias dos compostos de precipitação em função das categorias dos compostos de DIV (a) e TSM (b). Os círculos representam as categorias associados aos eventos de LGZ e os triângulos aos eventos de LPZ.

\section{AGRADECIMENTOS}

Este trabalho é uma parte da dissertação de Mestrado em Meteorologia do primeiro autor sob orientação do segundo. O trabalho foi realizado no Instituto Nacional de Pesquisas Espaciais (INPE) e apoiado pelo Departamento de Controle do Espaço Aéreo (DECEA), Instituto de Aeronáutica e Espaço (IAE) e Instituto de Controle do Espaço Aéreo (ICEA). Agradece-se também as valiosas sugestões feitas por dois revisores anônimos.

\section{APÊNDICE}

Detalham-se aqui alguns aspectos do método objetivo utilizado para estimar a posição, largura e precipitação média da ZCIT (PLPMZ). A área escolhida para identificar e estudar a ZCIT estende-se entre as latitudes $10^{\circ} \mathrm{S}$ e $18^{\circ} \mathrm{N}$ e a PLPMZ é obtida para a longitude de $27,5^{\circ} \mathrm{W}$ (Figura A1). Em testes realizados para longitudes mais próximas dos continentes $\left(22,5^{\circ} \mathrm{W}\right.$ e $\left.32,5^{\circ} \mathrm{W}\right)$, o método objetivo não apresentou bom desempenho, principalmente, nas posições mais austrais e boreais da ZCIT ao longo das longitudes de $32,5^{\circ} \mathrm{W}$ e $22,5^{\circ} \mathrm{W}$, respectivamente. Isso ocorreu devido à forte atuação de sistemas transientes de grande escala no hemisfério de verão que acaba influenciando a ZCIT Atlântica.

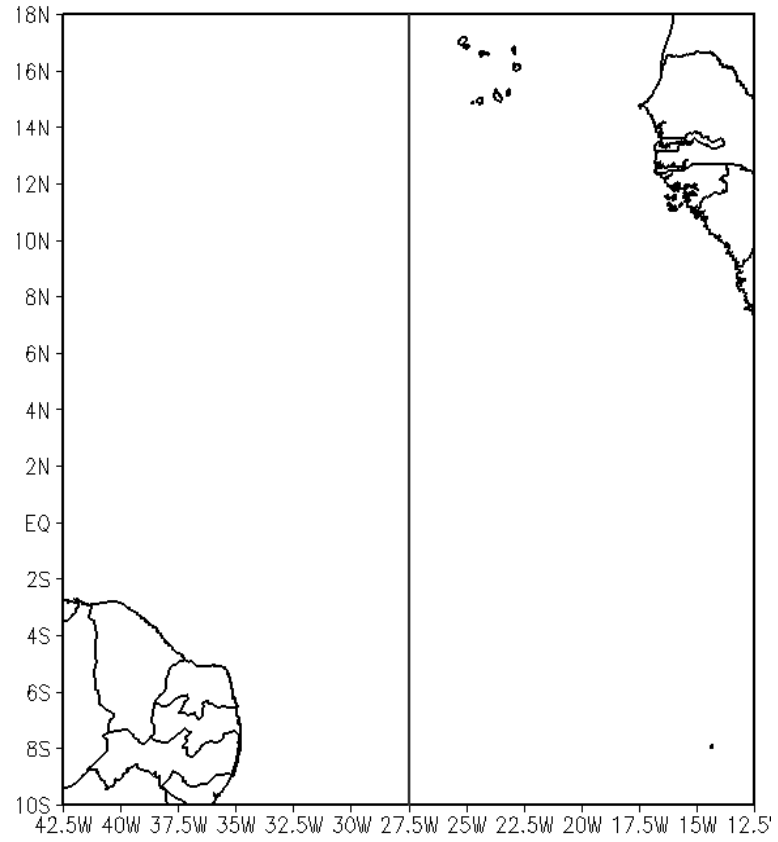

Figura A1 - Área de estudo. A linha contínua representa a longitude de $27,5^{\circ} \mathrm{W}$.

Inicialmente, são utilizadas duas séries de dados - dados diários de ROLE e de precipitação do GPCP - para obter o limiar de precipitação. Os dados de ROLE provêm do Climate Diagnosis Center do NOAA (CDC/NOAA) $)^{3}$ e encontram-se dispostos em uma grade regular com $2,5^{\circ}$ de espaçamento. Esses dados são utilizados para detectar a presença de nuvens convectivas, enquanto os do GPCP identificam regiões com altos índices de precipitação. As regiões sob nebulosidade convectiva (altos índices de precipitação), com valores abaixo (acima) do limiar de ROLE (precipitação), são utilizadas para representar a ZCIT.

Em estudos anteriores, diferentes limiares de ROLE foram propostos para a identificação de nuvens convectivas. Os valores dos limiares usualmente variaram entre $220 \mathrm{~W} . \mathrm{m}^{-2}$ a 240 W.m ${ }^{-2}$ (p. ex., Liebmann et al., 1999; Lau et al., 1997; Ferreira et al., 2005). Escolheu-se neste trabalho o limiar que apresenta a maior quantidade de valores válidos entre os limiares de 210 e $240 \mathrm{~W} \cdot \mathrm{m}^{-2}$. O valor escolhido foi de $230 \mathrm{~W} \cdot \mathrm{m}^{-2}$. Cabe ressaltar que limiares mais altos aumentam o número de pêntadas com nenhuma identificação de posição da ZCIT; mais baixos, com múltiplas posições ou com exagerada largura. $\mathrm{O}$ valor escolhido maximiza a quantidade de pêntadas com valores válidos, ou seja, minimiza a quantidade de erros (nenhuma ou múltiplas identificações, ou larguras irreais).

Para a precipitação, estudos indicam um limiar variando entre aproximadamente 5 a $10 \mathrm{~mm}$ (p. ex., Legates e Wilmott,

3. http://www.cdc.noaa.gov/cdc/data.intrep_ORL.htm 
1990; Adler et al., 2003; Chao, 2008; Chen et al., 2008). O limiar da precipitação foi estabelecido como o que maximiza o número de pêntadas com valores válidos e minimiza a diferença de largura (na escala mensal), quando se realiza a comparação com a largura estimada com os dados de ROLE. O limiar de precipitação escolhido foi de aproximadamente $7 \mathrm{~mm} \mathrm{~d}^{-1}$.

As estatísticas de comparação (ROLE $\times$ precipitação) consistem no erro médio (BE, Bias Error) ou viés e na raiz quadrada do erro quadrático médio (RMSE, Root Mean Square Error). O BE é dado por:

$$
B E=\frac{1}{N} \sum_{i=1}^{N}\left(L_{R_{i}}-L_{G_{i}}\right)
$$

que expressa a média da diferença entre a média mensal da largura estimada com os dados de $\operatorname{ROLE}\left(L_{R}\right)$ e a média mensal da largura estimada com dados de precipitação do $\operatorname{GPCP}\left(L_{G}\right)$, sendo $N=12$, i.e., número de meses do ano. O RMSE é dado por:

$$
R M S E=\sqrt{\frac{1}{N} \sum_{i=1}^{N}\left(L_{R_{i}}-L_{G_{i}}\right)^{2}}
$$

que é a raiz quadrada da média das diferenças individuais quadráticas entre $\mathrm{L}_{\mathrm{R}}$ e $\mathrm{L}_{\mathrm{G}}$.

Para o cálculo da posição latitudinal da ZCIT, no caso de precipitação, consideram-se os pontos cujo valor de precipitação seja maior do que o limiar, e o peso é a razão entre o limiar e o valor de precipitação (Equação 1). A expressão é a mesma utilizada no caso dos dados de ROLE.

Para uma dada longitude, ao atingir valores inferiores (superiores) ao limiar, a curva latitudinal de ROLE (precipitação) intercepta duas vezes a reta que representa o limiar, definindo dois pontos. A largura é definida como a distância entre esses dois pontos, ou melhor, a distância entre a lat ${ }_{i}$ mais ao norte e a lat $_{i}$ mais ao sul, que contém a posição e cujos valores de precipitação são maiores que o limiar durante uma determinada pêntada $\mathrm{k}$. Esses pontos são calculados por meio da interpolação linear entre os pontos de grade posterior e anterior à interceptação do limiar. As pêntadas que não atingem o limiar são identificadas por um valor indefinido (por exemplo, -999). Os casos onde a curva do gráfico cruza mais do que duas vezes o limiar são identificados por outro valor indefinido ( 999 , por exemplo). Estes casos podem estar associados a sistemas transientes (distúrbio ondulatório de leste, vórtice ciclônico de altos níveis), ZCIT dupla ou bifurcações da ZCIT, conforme os padrões na nebulosidade da ZCIT identificados por Coelho (2002). Com o objetivo de se obter uma maior quantidade de valores válidos para o estudo da variabilidade interanual da ZCIT, nos meses de março e abril, foco do estudo, os casos de valores indefinidos 999 contaminados por sistemas transientes são analisados individualmente. Nestes casos, a região de estudo tem sua extensão latitudinal diminuída para a região de atuação da ZCIT, i. e., a análise das variações dos dados é feita em uma região menor do que a inicialmente estabelecida, evitando a região de atuação do outro sistema, assim permitindo localizar univocamente a ZCIT. São também identificados por valor indefinido (888, por exemplo), valores estimados de largura maiores do que $10^{\circ}$. Geralmente, estes valores estão relacionados à atividade convectiva de outros sistemas atmosféricos acoplados à ZCIT.

A intensidade corresponde à precipitação média observada no intervalo de largura da ZCIT durante a pêntada k. A estimativa da precipitação média é obtida pelo método dos trapézios. Este método consiste em dividir a integral de uma função $f(x)$, definida no intervalo [a,b], em $n$ partes iguais e calcular a área do trapézio substituindo a curva em cada intervalo $x_{i-1}, x_{i}$ por uma reta ligando o pontos $f\left(x_{i-1}\right)$ e $f\left(x_{i}\right), i=1, \ldots, n$. $\mathrm{O}$ valor aproximado da precipitação média é a soma das áreas dos $n$ trapézios dividido pela largura.

\section{REFERÊNCIAS BIBLIOGRÁFICAS}

ADLER R. F.; HUFFMAN G. J.; CHANG, A.; FERRANO, R.; XIE, P.P.; JANOWIAK, J.; RUDOLF, B.; SCHNEIDER, U.; CURTIS, S.; BOLVIN, D.; GRUBER, A.; SUSSKIND, J., ARKIN, P.; NELKIN, E. The version-2 Global Precipitation Climatology Project (GPCP) monthly precipitation analysis (1979-present). Bulletin American Meteorological Society, v.4, p. 1147-1167, 2003.

BIASUTTI, M.; SOBEL, A. H.; KUSHNIR, Y. AGCM Precipitation Biases in Tropical Atlantic. Journal of Climate, v. 19, p. 935-958, 2006.

CHAO, W.C. The origins of ITCZs, monsoons and monsoon onset - A review. Submitted to Climate Dynamics, mar., 2008.

CHARNEY, J. G. Tropical cyclogenesis and the formation of Intertropical Convergence Zone. Mathematical Problems of Geophysical Fluids Dynamics, W. H. Reid, Ed., Lectures in Applied Mathematics. American Mathematical Society, v.13, p. 355-368, 1971.

CHEN, B.; LIN, X.; BACMEISTER, J.T. Frequency distribution of daily ITCZ patterns over the western-central Pacific. Journal of Climate, v. 21, n. 17, p. 4207-4222, 2008.

CHIANG, J. C. H.; KUSHNIR, Y.; GIANNINI, A. Deconstructing Atlantic Intertropical Convergence Zone variability: Influence of the local cross-equatorial sea surface temperature gradient and remote forcing from the eastern equatorial Pacific. Journal of Geophysical Research, v. 107, n. D1, 4004, 10.1029/2000JD000307, 2002.

COELHO, M. S. Estudo da estrutura vertical e horizontal da precipitação e da circulação atmosférica na região da 
ZCIT. 2002. 158 p. (INPE-10287-TDI/906). Dissertação (Mestrado em Meteorologia) - Instituto Nacional de Pesquisas Espaciais, São José dos Campos. 2002.

DAS, P. K. Monsoons. Fifth IMO lecture. Genebra, Suiça: World Meteorological Organization (WMO), n. 613, 1986, $155 \mathrm{p}$.

DE SOUZA, E. B.; KAYANO M. T.; AMBRIZZI, T. Intraseasonal and submonthly variability over the eastern Amazon and Northeast Brazil during the autumn rainy season. Theoretical and Applied Climatology, v. 81, p. 177-191, 2005.

FERREIRA, N. S. Zona de convergência intertropical. Boletim do Climanálise Especial - Comemoração dos 10 anos, São Paulo, 1996. Disponível em: http://climanalise.cptec.inpe.br/ $\sim$ rclimanl/boletim/cliesp10a/zcit_1. Acesso em: 28 fev. 2011.

FERREIRA, N. S.; REPELLI, C. A.; ALVEZ, J. M. B.; SOUZA, E. B. Loczcit um procedimento numérico para localização do eixo central da zona de convergência intertropical no atlântico tropical. Revista Brasileira de Meteorologia, v. 20, n. 2, p. 159-164, 2005.

FROM, L.D.; STAVER, A.E. Fundamentals of weather a workbook approach - Dubuque, Ia: Kendall/hunt, 1979. 30279507 ISBN 0-8403-2023.

GADGIL, S.; JOSEPH, P. V.; JOSHI, N. V. Ocean-atmosphere coupling over monsoon regions. Nature, v. 312, p. 141143, 1984.

GRAHAM, N. E.; BARNETT, T. P. Sea surface temperature, surface wind divergence and convection over tropical oceans. Science, v. 238, p. 657-659, 1987.

GU, G.; ADLER, R. F. Interannual variability of boreal summer rainfall in the equatorial Atlantic. International Journal of Climatology, v. 29, p. 175-184, 2009.

GUTZLER, D. S.; WOOD, T. M. Structure of large-scale convective anomalies over tropical oceans. Journal of Climate, v. 3, p. 483-496, 1990.

HASTENRATH, S.; HELLER, L. Dynamics of climatic hazards in Northeast Brazil. Quarterly Journal of the Royal Meteorological Society, v.103, n.435, p. 77-92, 1977.

HASTENRATH, S.; LAMB, P. Some aspects of circulation and climate over the eastern equatorial Atlantic. Monthly Weather Review, v.105, p. 1019-1023, 1977.

HASTENRATH, S. The relationship of highly reflective clouds to tropical climate anomalies. Journal of Climate, v.3, p. 353-365, 1990.

Hoffman, R. N.; Leidner S. M. An introduction to the near real time QuikSCAT data. Weather and Forecasting, v. 20, p. 476-493, 2005.

HOLTON, J.R.; WALLACE, J.M.; YOUNG, J. A. On boundary layer dynamics and the ITCZ. Journal of the Atmospheric Sciences, v. 28, p. 275-280, 1971.
HUFFMAN, G. J.; MORRISSEY, M., BOLVIN, D. T.; CURTIS, S.; JOYCE, R.; MCGAVOCK, B.; SUSSKIND, J. Global precipitation at one-degree daily resolution from multisatellite observations. Journal of Hydrometeorology, v. 2, p. 36-50, 2001.

KHRGIAN, A. Physical meteorology. In: Danilina, I.P. Meteorology and climatology - Boston, MA: G.K. Hall, 1977226674066 (Geophysics Series) ISBN I Summarie of Scientific Progress. Cap. 1, p. 1-18. ISBN 0-8161-2013-7.

KOUSKY, V. E. Pentad outgoing longwave radiation climatology for the South American sector. Revista Brasileira de Meteorologia, v. 3, p. 217-231, 1988.

KUMMEROW, C; BARNES, W; KOZU, T.; SHIUE, J.; SIMPSON, J. 1998: The Tropical Rainfall Measuring Mission (TRMM) sensor package. Journal of Atmospheric and Oceanic Technology, v. 15, p. 809-817, 1998.

LAU, K.-M.; WU, H.-T; BONY, S. The role of large-scale atmospheric circulation in the relationship between tropical convection and sea surface temperature. Journal of Climate, v. 10, p.381-392, 1997.

LEGATES, D. R.; WILLMOTT, C. J. Mean seasonal and spatial variability in gauge-corrected, global precipitation. International Journal of Climatology, v. 10, p. 111-127, 1990. LIEBMANN,B.;KILADIS,N.G.;MARENGO,J.A.;AMBRIZZI, T.; GLICK, J. D. Submonthly convective variability over South America and the south Atlantic convergence zone. Journal of Climate, v. 12, p. 1877-1891, 1999.

MELO, A. B. C.; CAVALCANTI, I. F. A., SOUZA, P. P. Zona de convergência intertropical do Atlântico. In: CAVALCANTI, I. F. A.; FERREIRA, N. J., SILVA, M. G. A. J.; DIAS, M. A. F. S. Tempo e clima no Brasil. São Paulo: Oficina de Textos, 2009. Cap. 2, p. 25-41. ISBN 978-85-86238-92-5.

MOLION, L. C. B.; BERNARDO, S. O. Uma Revisão da Dinâmica das Chuvas no Nordeste Brasileiro. Revista Brasileira de Meteorologia, v. 17, n. 1, p. 1-10, 2002.

MOURA, A. D.; SHUKLA, J. On the dynamics of droughts in northeast Brazil: observations, theory and numerical Experiments with a General Circulation Model. Journal of the Atmospheric Sciences, v. 38, n. 12, p. 2653-2675, 1981. NOBRE, P.; SHUKLA, J. Variations of sea surface temperature, wind stress and rainfall over the tropical Atlantic and South America. Journal of Climate, v.10, n. 4, p. 2464-2479, 1996.

PANOFSKY, H. A.; BRIER, G. W. Some applications of statistics to meteorology - University Park, PA: University Park, 1968.

PHILANDER, S. G. H.; GU, D.; HALPERN, D.; LAMBERT, G.; LAU, N. C.; Li, T.; PACANOWSKI, R. C. Why the ITCZ is mostly north of the equator. Journal of Climate, v. 9, p. 2958-2972, 1996. 
PIMENTEL, F. A.; FEREIRA, C. C. Técnicas para localização da ZCIT. Monitor Climático, v. 6, n. 66, p. 1-6, 1992.

SIMPSON, J.; ADLER, R. F.; NORTH, G.R. Proposed Tropical Rainfall Measuring Mission (TRMM) satellite. Bulletin American Meteorological Society, v. 69, p. 278-295, 1988.

SPIEGEL, M. R. Estatistica resumo da teoria: 875 problemas resolvidos, 619 problemas propostos Tradução de P. Cosentino - Sao Paulo, SP: Mcgraw-hill, 1979.

UVO, C. B. A Zona de Convergência Intertropical (ZCIT) e sua relação com a precipitação da Região Norte do Nordeste Brasileiro. 81p. (INPE - 4887 - TDL/378). Dissertação (Mestrado em Meteorologia) - Instituto Nacional de Pesquisas Espaciais, São José dos Campos, 1989.
WALISER, D.E., SOMMERVILLE, R.C. Preferred Latitudes for the Intertropical Convergence Zone. Journal of the Atmospheric Sciences., v. 51, n. 12, p. 1619-1639, 1994.

XIE, S.-P.; CARTON, J. A. Tropical Atlantic variability: Patterns, mechanisms, and impacts. Earth's Climate: The Ocean-Atmosphere Interaction, Geophysical Monograph, v. 147, p. 121-142, AGU, Washington, D. C, 2004. 